\title{
Pin-Align: A New Dynamic Programming Approach to Align Protein-Protein Interaction Networks
}

\author{
Farid Amir-Ghiasvand, Abbas Nowzari-Dalini, and Vida Momenzadeh \\ Department of Computer Science, School of Mathematics, Statistics, and Computer Science, College of Science, \\ University of Tehran, Tehran 1417614411, Iran \\ Correspondence should be addressed to Abbas Nowzari-Dalini; nowzari@ut.ac.ir
}

Received 16 June 2014; Accepted 15 October 2014; Published 10 November 2014

Academic Editor: Emil Alexov

Copyright (C) 2014 Farid Amir-Ghiasvand et al. This is an open access article distributed under the Creative Commons Attribution License, which permits unrestricted use, distribution, and reproduction in any medium, provided the original work is properly cited.

\begin{abstract}
To date, few tools for aligning protein-protein interaction networks have been suggested. These tools typically find conserved interaction patterns using various local or global alignment algorithms. However, the improvement of the speed, scalability, simplification, and accuracy of network alignment tools is still the target of new researches. In this paper, we introduce Pin-Align, a new tool for local alignment of protein-protein interaction networks. Pin-Align accuracy is tested on protein interaction networks from IntAct, DIP, and the Stanford Network Database and the results are compared with other well-known algorithms. It is shown that Pin-Align has higher sensitivity and specificity in terms of KEGG Ortholog groups.
\end{abstract}

\section{Introduction}

In the last decade, high throughput techniques in experimental biology produced a large amount of biological data which usually can be modeled by large networks such as metabolic networks, gene regulatory networks, and proteinprotein interaction (PPI) networks [1]. Analogous to biological sequence comparison [2-4], comparing large biological networks can improve our biological understanding of them. Comparison of PPI networks is a well-studied area due to the important roles of PPI networks. The main idea behind comparison of PPI networks is to find evolutionary conserved interaction modules which describes functional relevance. Exact comparison of large PPI networks is a NP-hard problem and there is no polynomial time algorithm for it. The NPhardness of this problem is based on the fact that this problem can be reduced to the subgraph isomorphism problem. In this way, the exact comparison of PPI networks is computationally infeasible, and PPI networks comparison is often addressed by heuristic methods [5-7].

Several algorithms have been proposed for biological network alignment. One of the first proposed network alignment algorithms is Path-Blast [8]. This algorithm finds highscore conserved pathways. After that, Sharan et al. designed
Network-Blast [9] in order to identify conserved protein complexes in multiple species. Koyuturk et al. devised an evolution-based scoring scheme to detect conserved clusters, called MaWISh (maximum weight induced subgraph) [10]. Flannick et al. proposed Graemlin as the first method to detect conserved subnetworks of arbitrary structures with progressive alignment method [11]. All these mentioned algorithms are called local alignment algorithms owing to the fact that they started to find subnetworks such as pathways and protein complexes and expanded their search results to obtain the feasible alignment. In local network alignment, each protein may be mapped to several proteins. On the other hand, global network alignment algorithm is defined to detect the best overall mapping across all parts of the input networks. IsoRank is the first global network alignment algorithm aiming to maximize the overall match between two networks [12]. Flannick et al. extended Graemlin to global network alignment by using a training set of known network alignments to learn parameters for the scoring function. This novel algorithm is called Graemlin 2.0 and is claimed to have linear time complexity with the number of PPIs [13]. The next version of IsoRank, IsoRankN [14], uses Nibble-Page-Rank algorithm [15] to align input networks locally and globally. Tian and Samatova introduced a connected-components 


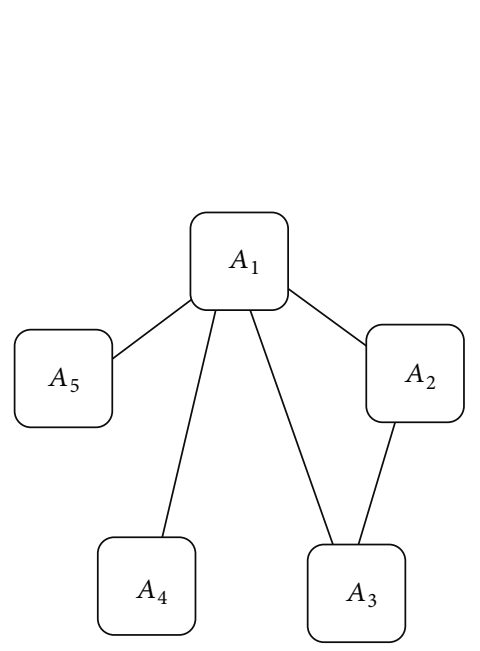

(a)

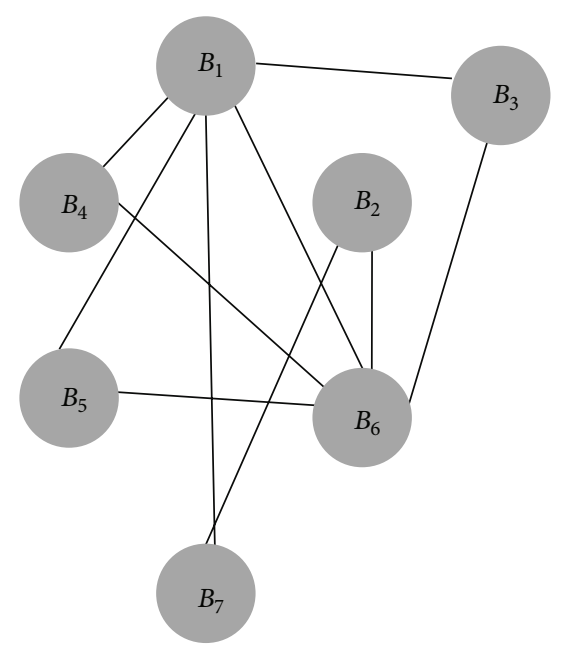

(b)

Figure 1: Two sample networks $A$ and $B$.

based algorithm, called HopeMap, for pairwise network alignment with the focus on fast identification of maximal conserved patterns [16]. After that, GRAAL [6] and $H$ GRAAL [1] are presented as the global network alignment algorithms based on seed-extend approaches and Hungarian algorithm, respectively. Both of GRAAL and $H-G R A A L$ algorithms are capable of aligning any kind of network owing to the fact that they are specifically designed based on network structure and do not use sequence similarity data. Observing that the size of true orthologs across species is small comparing to the total number of proteins in all species, Tian and Samatova presented a different approach based on a precompiled list of homologs identified by KO terms [17].

In this paper, Pin-Align (protein interaction network alignment) algorithm is introduced as a novel local network alignment algorithm for aligning two protein-protein interaction networks. In Pin-Align, Hub Clustering and hierarchical clustering algorithms are applied in the heuristic and dynamic programming phases in order to reduce time and space complexity of the problem. Two different types of scoring, Node-Scoring and Structural-Scoring, are used to find the best local alignments. Node-Scoring is simply based on BLAST bit score [18] and Structural-Scoring is based on the importance of Bridges (Bridges are considerable edges, which take part in most of the paths and consequently conserved paths in the networks) in biological networks.

The Pin-Align algorithm is performed on the protein interaction networks of Escherichia coli (E. coli), Salmonella typhimurium (S. typhimurium), Caulobacter crescentus (C. crescentus), human, mouse, yeast, and Drosophila melanogaste (fly) extracted from IntAct [19], DIP [20], and Stanford Network database (SNDB) [21]. The obtained results are compared with other well-known local alignment algorithms NetworkBLAST [9], MaWISh [10], Graemlin [11], and Graemlin 2.0 [13], in a way that Graemlin 2.0 compares its results, because it is the only method that compares results of all local alignment tools against KEGG Ortholog (KO) groups [22].

\section{Materials and Methods}

Protein-protein interaction (PPI) network is defined as the set of relationships among proteins. Here, a PPI network is modeled by an undirected and weighted graph $G=(V, E)$, where nodes correspond to proteins and each weighted edge specifies the probability that two proteins interact. For two graphs $G_{1}=\left(V_{1}, E_{1}\right)$ and $G_{2}=\left(V_{2}, E_{2}\right)$ (such as two sample networks $A$ and $B$ in Figure 1), the goal of network alignment is to identify possible mappings which map vertices of $G_{1}$ into vertices of $G_{2}$.

In addition, for each mapping, the corresponding set of conserved edges is also identified. Mappings may be partial; that is, they do not need to be defined for all the nodes of the graphs. Each mapping implies a common subgraph between the two graphs. When protein $a_{1}$ from graph $G_{1}$ is mapped to protein $a_{2}$ from graph $G_{2}$, then $a_{1}$ and $a_{2}$ refer to the same node in the common subgraph; the edges in the common subgraph correspond to the conserved edges [23].

Pin-Align algorithm is a local network alignment algorithm, mainly designed based on the dynamic programming approach. To explain Pin-Align in more detail, first a formal definition of local network alignment is presented. Given two graphs $G_{1}\left(V_{1}, E_{1}\right)$ and $G_{2}\left(V_{2}, E_{2}\right)$, local alignment $\mathrm{LA}_{G_{1}, G_{2}}\left(\mathrm{Sg}_{1}, \mathrm{Sg}_{2}, M\right)$ is a triplet where $\mathrm{Sg}_{1}$ and $\mathrm{Sg}_{2}$ are the subgraphs of $G_{1}$ and $G_{2}$, respectively, and $M$ is defined as the mapping which aligns vertices of $\mathrm{Sg}_{1}$ into vertices of $\mathrm{Sg}_{2}$. Two sample local alignments of networks $A$ and $B$ of Figure 1 are shown in Figure 2(a).

Following, the best local network alignment (BLA) is defined as a function to obtain the best local alignment 


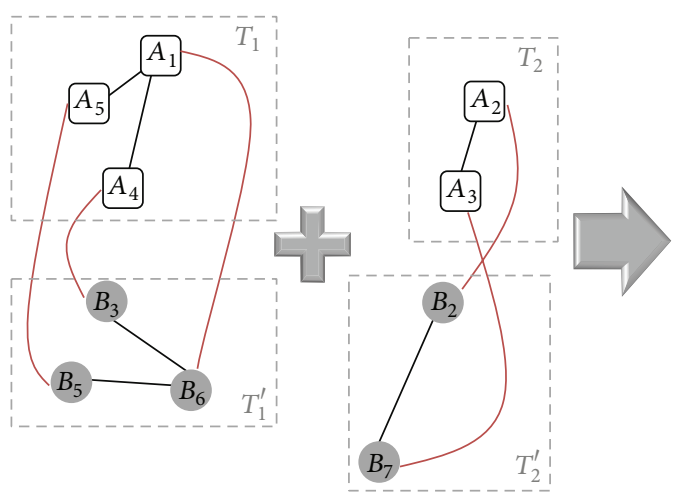

(a)

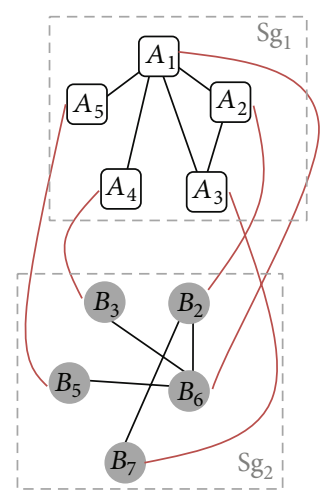

(b)

Figure 2: (a) Two local alignments of networks $A$ and $B$ of Figure 1. (b) Result of merging them.

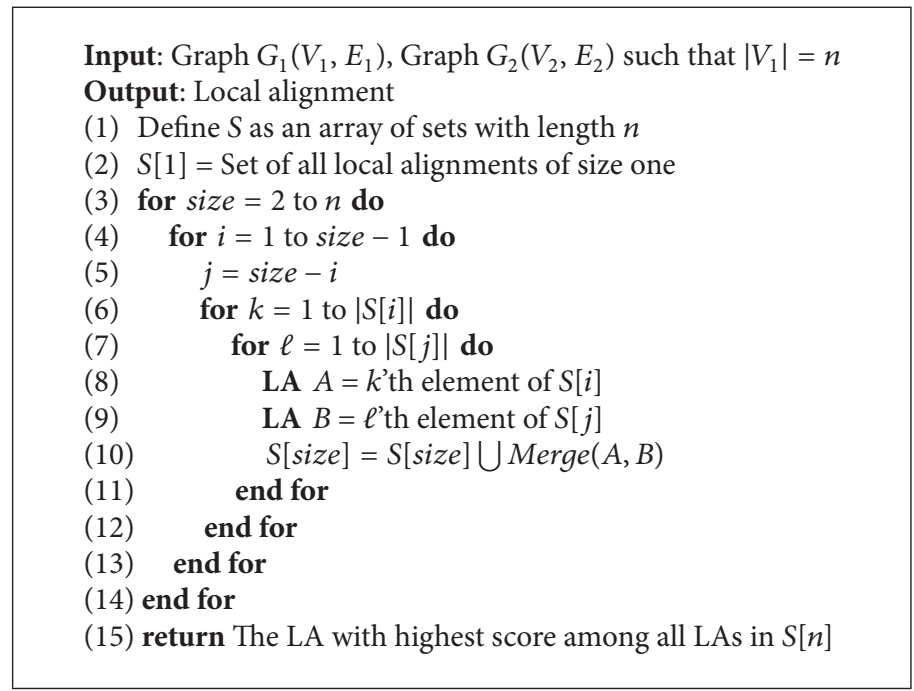

Algorithm 1: Simple-Align algorithm.

for each subgraph of $G_{1}$. The best local alignment of $\mathrm{Sg}_{1}$ as a subgraph of $G_{1}$ with a subgraph of $G_{2}$ is shown by BLA $_{G_{1}, G_{2}}\left(\mathrm{Sg}_{1}\right)$, which represents a local alignment with the highest score among all possible alignments between $\mathrm{Sg}_{1}$ and all possible subgraphs of $G_{2}$ that can be mapped to $\mathrm{Sg}_{1}$.

Now, a naive dynamic programming algorithm for network alignment is demonstrated in the next subsection, and later Pin-Align algorithm is presented.

2.1. Dynamic Programming Algorithm to Align PPI Networks. In alignment of two weighted graphs $G_{1}$ and $G_{2}$ through dynamic programming technique, we generate all LAs of size one (subgraphs with a single vertex) in the first step. For obtaining LAs of larger subgraphs of $G_{1}$, we merge all possible pairs of LA of smaller subgraphs. In other words, for each subgraph of $G_{1}$ such as $\mathrm{Sg}_{1}$, there are several pairs of distinct subgraphs (subgraphs with no common vertices) such as $\left(T_{1}, T_{2}\right)$ where $\mathrm{Sg}_{1}=T_{1} \cup T_{2}$. Figure 2 demonstrated the merge process of two sample local alignments of networks $A$ and $B$ of Figure 1 . We produce all possible local alignments for
$\mathrm{Sg}_{1}$ such as $\mathrm{LA}_{G_{1}, G_{2}}\left(\mathrm{Sg}_{1}, \mathrm{Sg}_{2}, M\right)$ by merging every possible local alignments of $T_{1}$ such as $\mathrm{LA}_{G_{1}, G_{2}}\left(T_{1}, T_{1}^{\prime}, M^{\prime}\right)$ with every possible local alignment of $T_{2}$ such as $\mathrm{LA}_{G_{1}, G_{2}}\left(T_{2}, T_{2}^{\prime}, M^{\prime \prime}\right)$. This process is done iteratively, and finally all local alignments of $G_{1}$ including $\mathrm{BLA}_{G_{1}, G_{2}}\left(G_{1}\right)$ are obtained.

Inspired by dynamic programming technique, we propose Simple-Align algorithm in Algorithm 1. This algorithm can find all BLAs; however by considering time and memory complexity, this algorithm is not feasible. To overcome this, we use several heuristics to reduce memory and time complexity and propose a new local alignment algorithm, Pin-Align, in next section.

2.2. Pin-Align Algorithm. As explained, Pin-Align uses dynamic programming approach to solve the local network alignment problem and overcome the deficiencies of dynamic programming such as time and space complexity by applying heuristic approach. The steps of the Pin-Align algorithm are summarized as follows. 
(1) Partition the input graph $G_{1}$ into smaller clusters. These clusters are dense subgraphs of $G_{1}$. The partitioning is done by using a new clustering algorithm named Hub Clustering. Clusters obtained in this step are named $\mathrm{Hub}$-Clusters.

(2) For each Hub-Cluster,

(a) create local alignments of size one with BLAST bit score greater than 0 , between vertices of $\mathrm{Hub}_{\text {-Cluster }}$ and vertices of $\mathrm{G}_{2}$; local alignments obtained by this approach are named Candidates $\left(C=\mathrm{LA}_{G_{1}, G_{2}}\left(\mathrm{Sg}_{1}, \mathrm{Sg}_{2}, M\right)\right.$ such that $\mathrm{Sg}_{1}$ is a subgraph of $\mathrm{Hub}$-Cluster ${ }_{i}$ with size one),

(b) collect all Candidates of subgraph $\mathrm{Sg}_{1}$ of $G_{1}$ (such as $C=\mathrm{LA}_{G_{1}, G_{2}}\left(\mathrm{Sg}_{1}, \mathrm{Sg}_{2}, M\right)$ ) in a new set called Candidate-Collection and each Candidate-Collection is represented by its subgraph of $G_{1}$ such as $\mathrm{CC}\left(\mathrm{Sg}_{1}\right)$; Candidate-Collections obtained for single nodes of $G_{1}$ are used as seeds in Step 2(c),

(c) merge small Candidate-Collections (seeds) to gain larger ones. Repeat this process continuously to achieve a Candidate-Collection $\mathrm{CC}\left(\mathrm{Hub}\right.$-Cluster $\left.{ }_{i}\right)$ as the final result of merging process. Size of a Candidate or a CandidateCollection is defined by the number of vertices of its subgraph of $G_{1}$ and cardinality of a Candidate-Collection is defined as the number of its Candidates. In this step, each Candidate-Collection can be merged with several Candidate-Collections and create CandidateCollections with larger size. For determining which Candidate-Collections should be merged together, a new hierarchical clustering is used as a pattern of merging.

The Candidate-Collections of Hub-Clusters are named Final-Candidate-Collections.

(3) Based on Final-Candidate-Collections, find final alignment using similarity graph. This graph is a special weighted bipartite graph where first and second set of vertices of it are shown by $V_{1}$ and $V_{2}$ which are the set of vertices of $G_{1}$ and $G_{2}$. The weight of each edge is computed based on Final-CandidateCollections.

Flowchart of Pin-Align algorithm is presented in Figure 3 and its details are explained by an example on two networks $A$ and $B$ given in Figure 1 .

In Step 1, in order to reduce the search space of the problem, input graph $G_{1}=\left(V_{1}, E_{1}\right)$ is clustered into some dense subgraphs and Step 2 of the algorithm runs for each dense subgraph separately. Generally PPI networks are sparse graphs, but they have dense regions containing high degree vertices named hubs. These regions usually contain protein complexes; therefore conserved modules among different PPIs usually exist in these regions.

To detect dense subgraphs in the graph $G_{1}$, a novel clustering algorithm, called Hub Clustering, is proposed. The

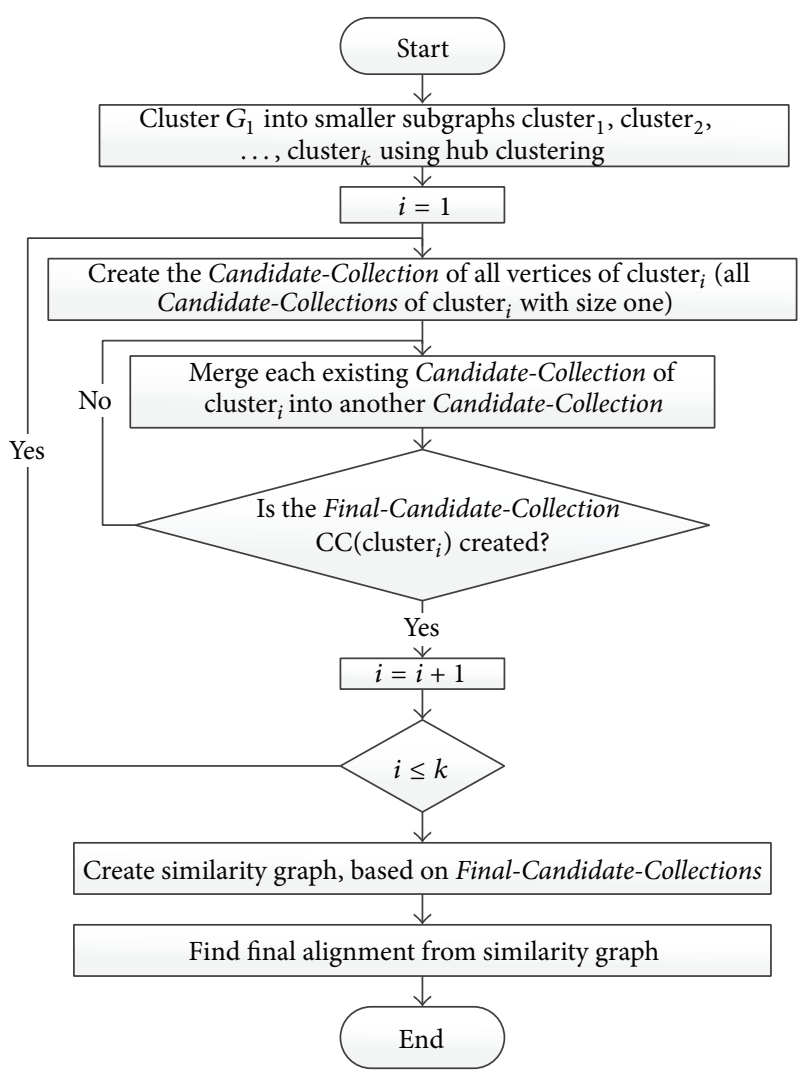

Figure 3: Flowchart of Pin-Align algorithm.

importance of hub nodes and density of subgraphs are two major criteria for this clustering method. First, a portion of highly connected vertices are selected as hub nodes. The vertices degree of these nodes is greater than $95 \%$ of other nodes. The hub nodes are considered as the center vertices of dense regions of graph $G_{1}$ and are supposed to be initial clusters. For other nodes of $G_{1}$, each node is joint to the nearest cluster (the cluster that contains its nearest hub). For this aim, the length of each edge is defined as the negative logarithm of its weight (from adjacency matrix), and then Dijkstra shortest path algorithm [24] is used to find the nearest cluster. The obtained clusters are named $\mathrm{Hub}$-Clusters.

In Step 2, each of Hub-Clusters of $G_{1}$ (obtained in Step 1) is aligned to $G_{2}$. As mentioned, Step 2(a) creates Candidates of size one based on BLAST bit score of vertices of two graphs $G_{1}$ and $G_{2}$ (BLAST bit score is a normalized score which is calculated by basic local alignment search tool, based on sequence similarity [18]). Figure 4(a) shows BLAST bit scores of proteins of networks $A$ and $B$.

After that, all Candidates of each vertex of $G_{1}$ are collected into a Candidate-Collection with size one as a seed in Step 2(b). In other words, Candidate-Collection of each vertex of $G_{1}$ such as $\operatorname{Sg}_{1}$ contains all Candidates for $\operatorname{Sg}_{1}$ from $G_{2}$ which can be aligned with $\mathrm{Sg}_{1}$. For example, Candidates of networks $A$ and $B$ of Figure 1 are shown in Figure 4(b) and those Candidate-Collections are shown in Figure 4(c).

In Step 2(c), two seeds (i.e., Candidate-Collections $\mathrm{CC}\left(\mathrm{Sg}_{i}\right)$ and $\left.\mathrm{CC}\left(\mathrm{Sg}_{j}\right)\right)$ are merged in order to produce a 


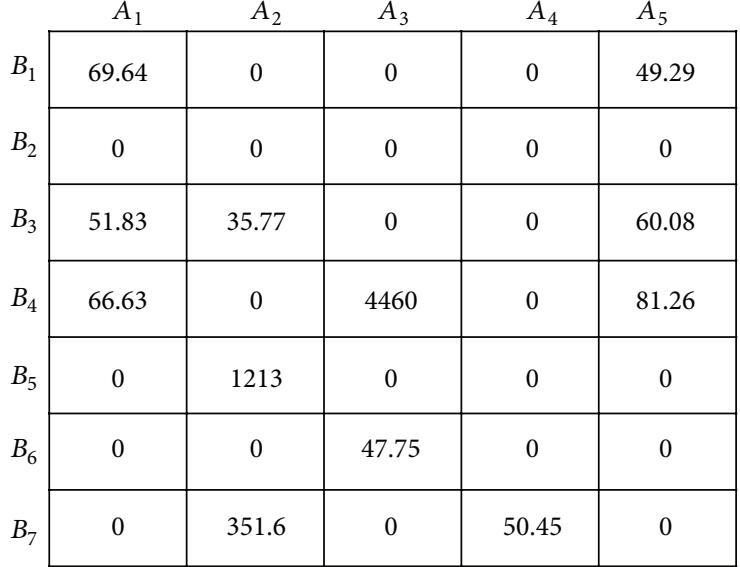

(a)

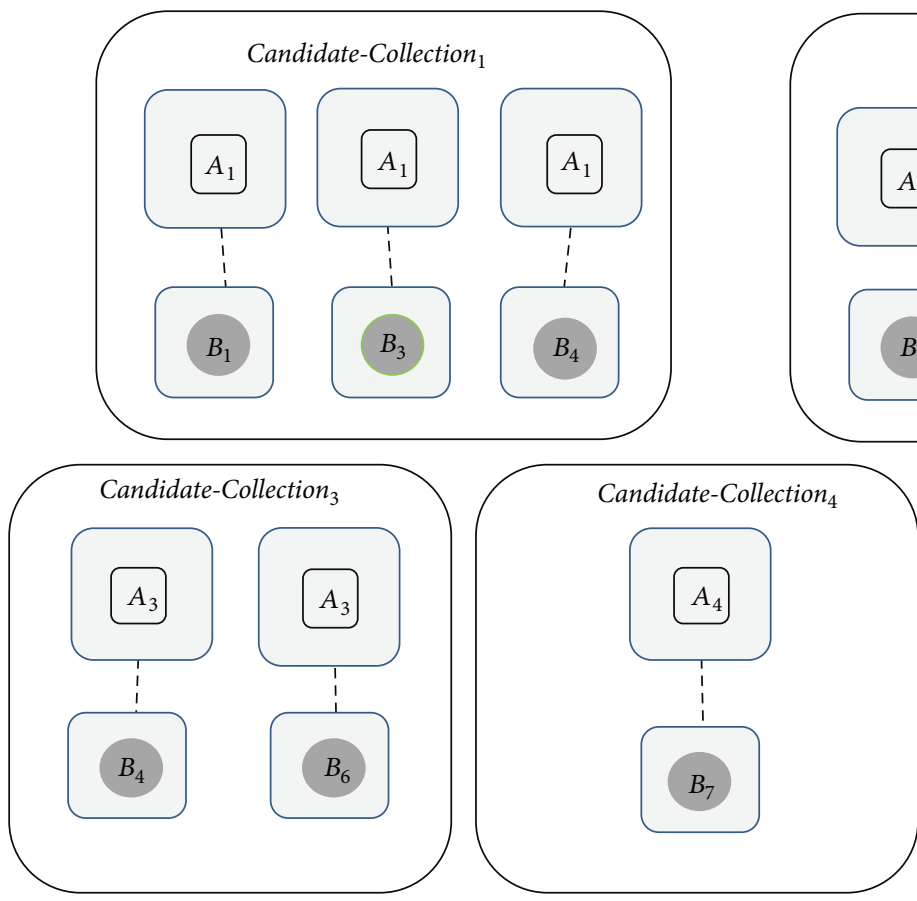

(c)

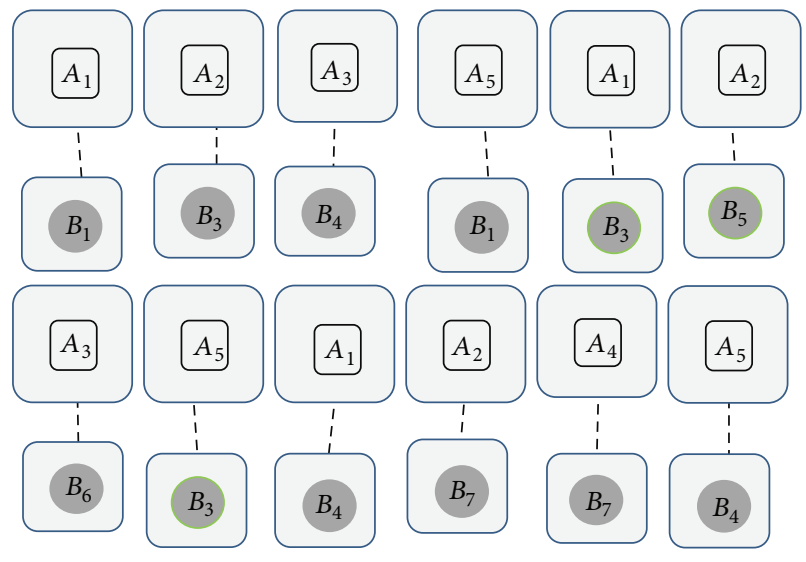

(b) 

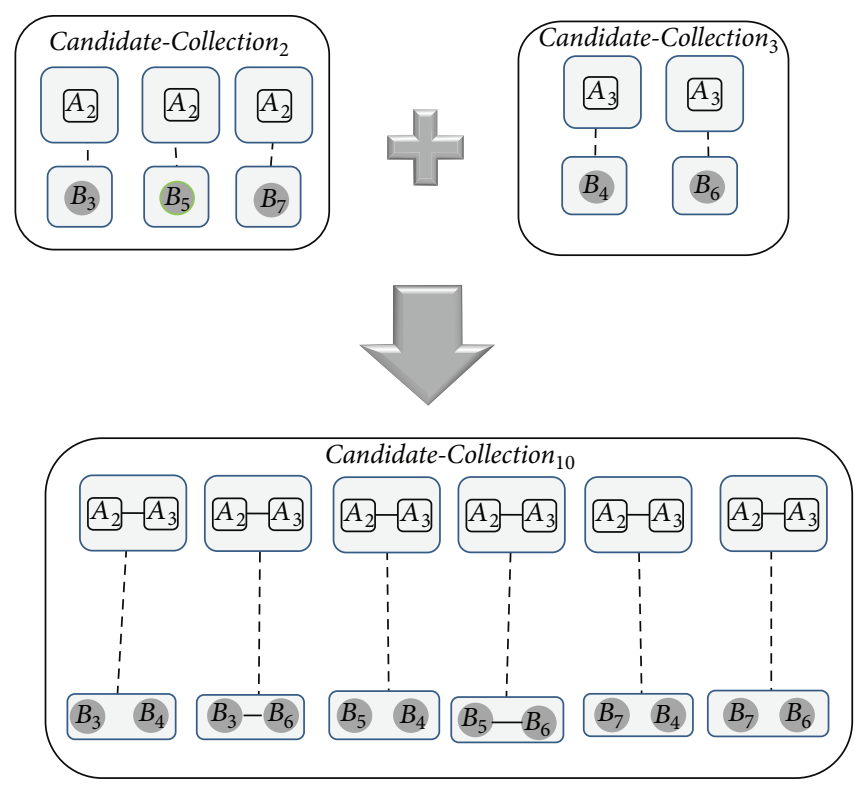

Figure 5: Two Candidate-Collections and the result of merging them.

pattern for merging Candidate-Collections. Cardinality of new Candidate-Collections obtained in higher levels of hierarchical clustering tree is increased exponentially as a result of merging smaller Candidate-Collections, so we have to save the Candidates with highest score and discard Candidates with low scores. In Step 2(c) we use a multiobjective optimization technique for finding the highest score Candidates [26]. The highest score Candidates are obtained by sorting Candidates based on two criteria, first, Nodes-Scores; next, StructuralScores. Finally, the Candidates with the highest scores are chosen in each step. Following, we describe this scoring schema.

Node-Score. Node-score of each Candidate $C=\operatorname{LA}_{G_{1}, G_{2}}\left(\mathrm{Sg}_{1}\right.$, $\left.\mathrm{Sg}_{2}, M\right)$ is obtained by using the following equation:

$$
\begin{aligned}
& \text { NodeScore }(C) \\
& =\sum_{i=1}^{\left|\mathrm{Sg}_{1}\right|} \text { BLASTBitScore }\left(\mathrm{Sg}_{1}^{i}, \mathrm{MV}\left(\mathrm{Sg}_{1}^{i}, M\right)\right) .
\end{aligned}
$$

In this equation, $i$ is an iterator on vertices of $S g_{1}$ and $S g_{1}^{i}$ is the $i$ th vertex of $\mathrm{Sg}_{1} ; \mathrm{MV}(v, M)$ indicates a vertex of $\mathrm{Sg}_{2}$ mapped into $v \in \mathrm{Sg}_{1}$ by the mapping $M$.

Structural-Score. Due to the existence of hubs and small words in biological networks, certainly the network contains bridges. Bridges are considerable edges, which take part in most of the paths (specifically conserved paths) in the networks. Because of the importance of bridges, if $\mathrm{Sg}_{1}=$ $\left(V_{1}^{\prime}, E_{1}^{\prime}\right)$ is a subgraph of $G_{1}=\left(V_{1}, E_{1}\right)$ and $\operatorname{Sg}_{2}=\left(V_{2}^{\prime}, E_{2}^{\prime}\right)$ is a subgraph of $G_{2}=\left(V_{2}, E_{2}\right)$, the structural scoring function for each Candidate $C=\mathrm{LA}_{G_{1}, G_{2}}\left(\mathrm{Sg}_{1}, \mathrm{Sg}_{2}, M\right)$ is defined as follows:

$$
\begin{aligned}
& \text { StructuralScore }(C) \\
& =\sum_{v \in V_{1}^{\prime}} \sum_{u \in V_{1}^{\prime}}(f(v) \times f(u))^{\alpha} \\
& \quad \times\left(P_{G_{1}}(v, u) P_{G_{2}}(\operatorname{MV}(v, M),\right. \\
& \qquad \operatorname{MV}(u, M)))^{1-\alpha} .
\end{aligned}
$$

In this equation, $f(i)$ shows the number of conserved edges at vertex $i$ in the subgraph $S_{1} ; \alpha$ is a constant value between 0 and 1 and shows the importance of adjacent vertices degrees; $P_{G}(x, y)$ is the probability of interaction between protein $x$ and protein $y$ in the network $\mathrm{G}$.

As mentioned before, to control the exponential growth of the cardinality of Candidate-Collections in combining process, we use a multiobjective optimization method on node score and structural score, as optimization objectives. In this way, after creating each Candidate-Collection among all Candidates obtained in that, we select only a limited number of Candidates (based on upon scoring functions) and discard others. Obtained Candidate-Collections of Hub-Clusters are named Final-Candidate-Collections which contain FinalCandidates (Candidates of Hub-Clusters).

After creating all Final-Candidate-Collections, in Step 3 of Pin-Align Algorithm, an Initial Similarity Bipartite Graph $\operatorname{ISBG}\left(G_{1}, G_{2}\right)=\left(V_{1}, V_{2}, E\right)$ is created which consists of vertices of $G_{1}=\left(V_{1}, E_{1}\right)$ as its first part and vertices of $G_{2}=$ $\left(V_{2}, E_{2}\right)$ as its second part, and $E$ is a set of weighted edges between $V_{1}$ and $V_{2}$. The weight of each edge represents the similarity between its incident vertices. Let $C\left(v_{i}, u_{j}\right)$ be a set of all Final-Candidates which maps $v_{i}$ into $u_{j}$. In $\operatorname{ISBG}\left(G_{1}, G_{2}\right)$, 


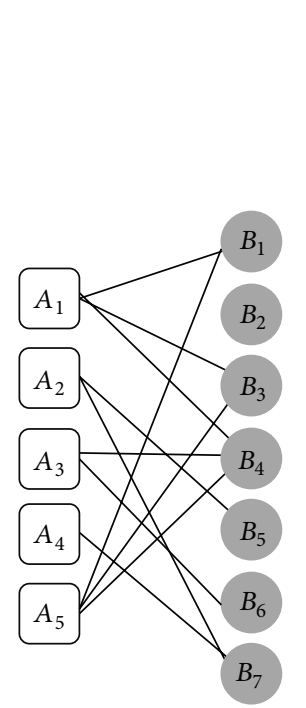

(a)

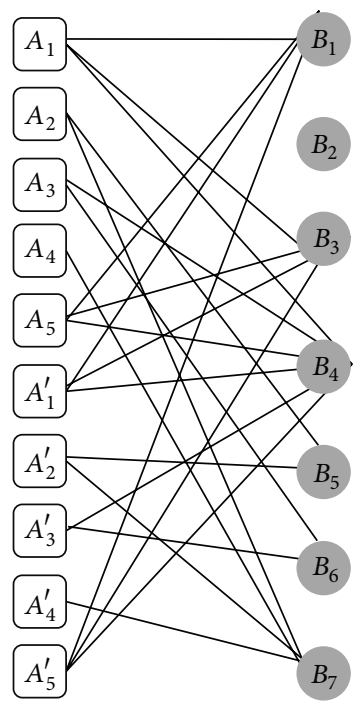

(b)

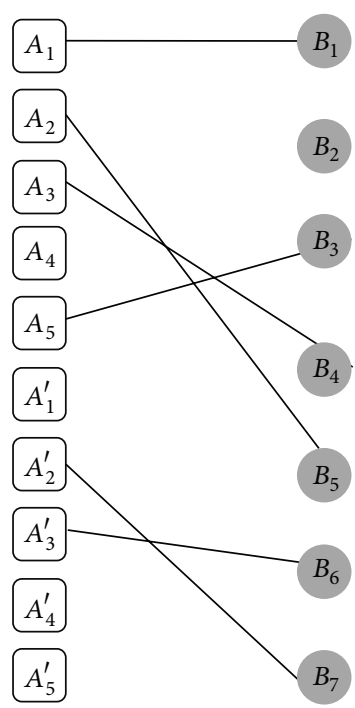

(c)

Figure 6: (a) The Initial Similarity Bipartite Graph (ISBG) between networks $A$ and $B$ in Figure 1 (the weights of edges are hidden for simplicity). (b) The ISBG, with duplication of first part. (c) Graph $M_{1}$, obtained from first part of duplicated ISBG by performing Hungarian algorithm.

the weight of an edge $e=\left\{v_{i}, u_{j}\right\}$, where $v_{i} \in V_{1}$ and $u_{j} \in V_{2}$, is equal to zero if $\left|C\left(v_{i}, u_{j}\right)\right|=0$; otherwise this weight is defined by the following formula:

$$
\begin{aligned}
W\left(v_{i}, u_{j}\right)= & \left(\operatorname{Node}_{\operatorname{Sim}}\left(v_{i}, u_{j}\right)+\text { Neighborhoods }_{\operatorname{Sim}}\left(v_{i}, u_{j}\right)\right) \\
& \times \frac{1}{\left|C\left(v_{i}, u_{j}\right)\right|},
\end{aligned}
$$

where $\operatorname{Node}_{\operatorname{Sim}}\left(v_{i}, u_{j}\right)$ is the similarity between two proteins $v_{i}$ and $u_{j}$ and it is calculated by using the following formula:

$$
\operatorname{Node}_{\operatorname{Sim}}\left(v_{i}, u_{j}\right)=\operatorname{BLASTBitScore}\left(v_{i}, u_{j}\right) \times\left|C\left(v_{i}, u_{j}\right)\right| \text {, }
$$

Neighborhoods $\operatorname{Sim}_{\text {Sim }}\left(v_{i}, u_{j}\right)$ is the similarity between conserved neighborhoods of $v_{i}$ and $u_{j}$ and is calculated as follows:

$$
\begin{aligned}
& \text { Neighborhoods }_{\operatorname{Sim}}\left(v_{i}, u_{j}\right) \\
& =\sum_{c \in C\left(v_{i}, u_{j}\right)} \sum_{\left(v_{i}^{\prime}, u_{j}^{\prime}\right) \in \mathrm{CN}_{c}\left(v_{i}, u_{j}\right)} \text { BLASTBitScore }\left(v_{i}^{\prime}, u_{j}^{\prime}\right) .
\end{aligned}
$$

Let $N_{G}(v)$ be the set of all neighbors of $v\left(N_{G}(v)=\{u \in\right.$ $V(G) \mid u v \in E(G)\})$; then $\mathrm{CN}_{c}\left(v_{i}, u_{j}\right)$ for Candidate $c=$ $\mathrm{LA}_{G_{1}, G_{2}}\left(\mathrm{Sg}_{1}, \mathrm{Sg}_{2}, M\right)$ is

$$
\mathrm{CN}_{c}\left(v_{i}, u_{j}\right)=\left\{\left(v_{i}^{\prime}, \operatorname{MV}\left(v_{i}^{\prime}, M\right)\right) \mid v_{i}^{\prime} \in N_{G_{1}}\left(v_{i}\right)\right\} .
$$

An Initial Similarity Bipartite Graph for networks $A$ and $B$ in Figure 1 is shown in Figure 6(a). After creating the Initial Similarity Bipartite Graph (ISBG), we can find just a single mapping for each vertex by finding maximum matching in this graph. The maximum matching can be found by applying the Hungarian algorithm (with polynomial time complexity) on the ISBG [27].

As mentioned, this approach can find just a single mapping for each vertex, although it cannot support some evolutionary functions such as duplication and diversion. To support duplication and diversion, we should consider matching of a single vertex from one part into two vertices of other parts in the ISBG. This type of matching is named DMatch. In other words, DMatchs are paths with length 2 such as $P\left(v_{i}^{\prime}, v_{j}, v_{k}^{\prime}\right)$ in the ISBG which aligns $v_{j}$ from one graph into both $v_{i}^{\prime}$ and $v_{i}^{\prime}$ from another graph. Here the problem is to find a set of Matchs and DMatchs from the ISBG where sum of their weights is maximum. This problem is named Maximum Weighted DMatching. To decrease the size of the solution space of this problem, we decrease the size of edge set of the ISBG by deleting some edges. The above procedure is performed by Algorithms 2 and 3.

Algorithm 2 gives the ISBG as input and creates the Final Similarity Bipartite Graph. In Steps 1-4 of Algorithm 2 all vertices of one part of the ISBG are duplicated and each duplicated vertex is connected to all vertices conjunct to the main vertex with the same weight as shown in Figure 6(b); then the Hungarian algorithm is performed on this graph in Step 5 of Algorithm 2. Obtained bipartite graph is named $M_{1}$. The result of these steps for ISBG of Figure 6(a) is shown in Figure 6(c). All duplicated vertices of $M_{1}$ are merged together in Step 6 of Algorithm 2. In Steps 7-12 of Algorithm 2, the same process is implemented on the other part of the obtained ISBG and the matching $M_{2}$ is obtained. Figures 7(a) and 7(b) illustrate these steps for ISBG of Figure 6(a). Step 13 of Algorithm 2 combines $M_{1}$ and $M_{2}$ to create $\operatorname{Sim}_{G}$ as the Final Similarity Bipartite Braph as shown in Figure 7(c). 
Input: Initial-similarity-bipartite-graph $S=\left(S_{1}, S_{2}, E_{S}\right)$ Output: Graph $\operatorname{Sim}_{G}$

(1) Bipartite-graph $S D_{1}=S$

(2) for all vertex $v \in S_{1}$ do

(3) Duplicate $v$ in $S D_{1}$

(4) end for

(5) Bipartite-graph $M_{1}=$ Maximum-Weight-Matching $\left(S D_{1}\right)$

(6) Merge duplicated vertices of $S D_{1}$ in $M_{1}$

(7) Bipartite-graph $\mathrm{SD}_{2}=S$

(8) for all vertex $v \in S_{2}$ do

(9) Duplicate $v$ in $S D_{2}$

(10) end for

(11) Bipartite-graph $M_{2}=$ Maximum-Weight-Matching $\left(S D_{2}\right)$

(12) Merge duplicated vertices of $S D_{2}$ in $M_{2}$

(13) Bipartite-graph $\operatorname{Sim}_{G}=M_{1} \cup M_{2}$

(14) return $\operatorname{Sim}_{G}$

Algorithm 2: Find $\operatorname{Sim}_{G}$ Graph.

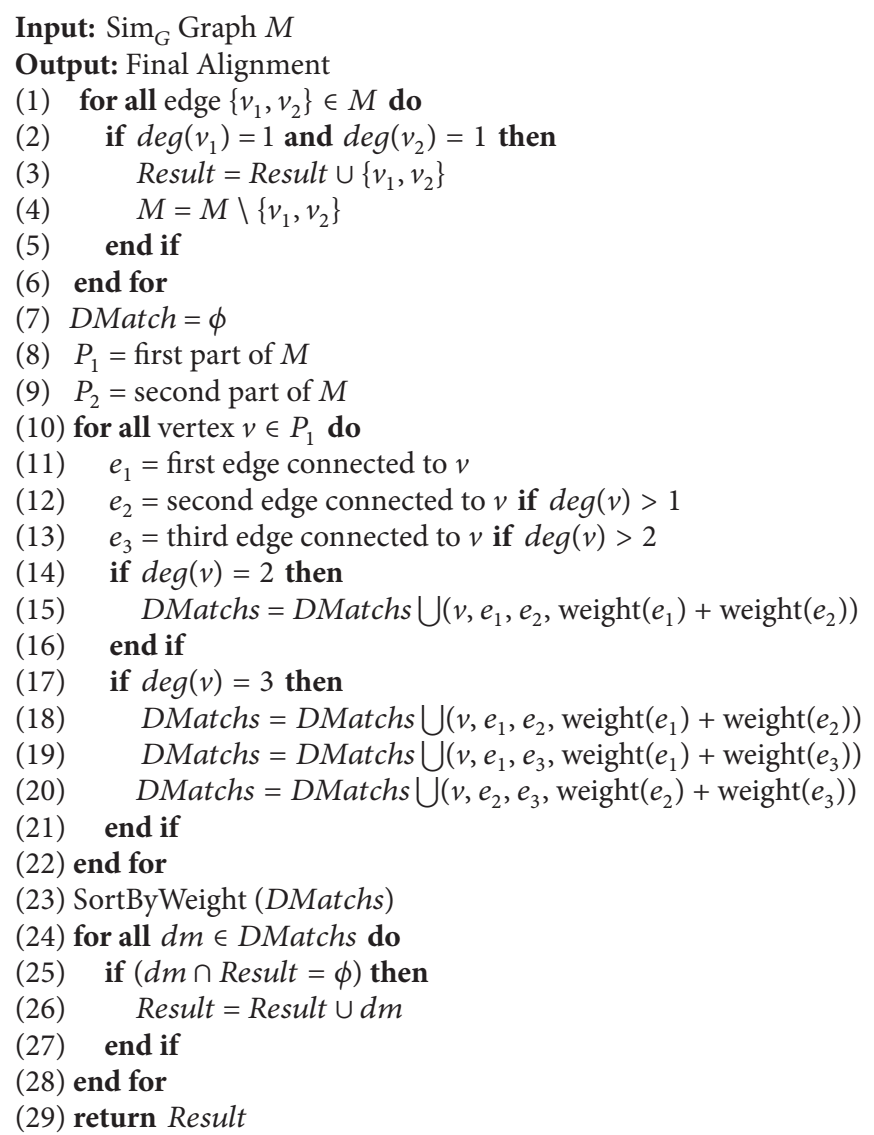

Algorithm 3: Find final alignment.

It can be proven that Maximum Weighted Dmatchings of graph $\operatorname{Sim}_{G}$ are exactly similar to Maximum Weighted Dmatchings of ISBG. So $\operatorname{Sim}_{G}$ contains all edges of Maximum Weighted Dmatching and the degree of its vertices is at most 3. For this reason obviously the solution space of the Maximum Weighted DMatching problem on $\operatorname{Sim}_{G}$ is considerably decreased in comparison with the ISBG.

To find the final alignment using the graph $\operatorname{Sim}_{G}$, we use a greedy algorithm with polynomial time complexity as shown in Algorithm 3. Algorithm 3 gives the Final Similarity 


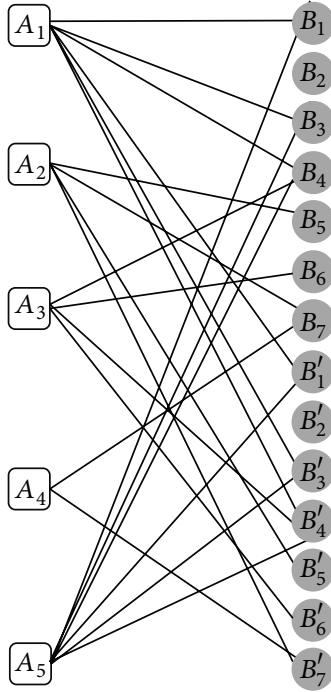

(a)

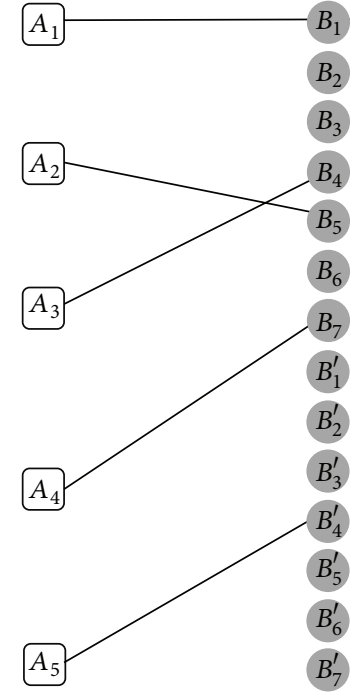

(b)

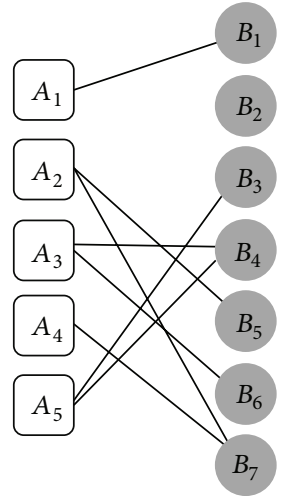

(c)

Figure 7: (a) The ISBG between networks $A$ and $B$ in Figure 1, with duplication of second part. (b) Graph $M_{2}$, obtained from second part duplicated ISBG by performing Hungarian algorithm. (c) The Final Similarity Bipartite Graph ( $\operatorname{sim}_{G}$ ) for networks $A$ and $B$.

Bipartite Graph $\operatorname{Sim}_{G}$ (obtained by Algorithm 2) as input and matches all pairs of adjacent vertices where degree of both of them is one. For other vertices, Algorithm 3 creates all possible Dmatchs and selects them in a greedy manner. Algorithm 3 utilizes the following steps for all vertices of graph $\operatorname{Sim}_{G}$.

(1) If the degree of the vertex $v$ is one and degree of adjacent vertex is also one, these two vertices are assigned to each other.

(2) If the degree of the vertex $v$ is two and $v$ is adjacent to $v_{i}^{\prime}$ and $v_{j}^{\prime}$, the path $P\left(v_{i}^{\prime}, v, v_{j}^{\prime}\right)$ is created as a potential Dmatch.

(3) If the degree of the vertex $v$ is three and $v$ is adjacent to $v_{i}^{\prime}, v_{j}^{\prime}$, and $v_{k}^{\prime}$, potential Dmatchs $P\left(v_{i}^{\prime}, v, v_{j}^{\prime}\right)$, $P\left(v_{i}^{\prime}, v, v_{k}^{\prime}\right)$, and $P\left(v_{j}^{\prime}, v, v_{k}^{\prime}\right)$ are created.

The weight of a given DMatch can be calculated by the following formula:

$$
W\left(P\left(v_{i}^{\prime}, v, v_{j}^{\prime}\right)\right)=W\left(v_{i}, v\right)+W\left(v, v_{j}\right) .
$$

After creating all potential DMatchs, we sort them based on their weights; then in a successive rounds we choose the highest weight DMatchs $P\left(v_{i}^{\prime}, v, v_{j}^{\prime}\right)$ and delete all other edges connected to $v_{i}^{\prime}, v$, or $v_{j}^{\prime}$. By this way all assignments are found and finally the local alignment of two graphs is obtained in Steps 24-27 of Algorithm 3. Therefore the output of this algorithm is the final alignment between two given graphs $G_{1}$ and $G_{2}$. The final alignment between networks $A$ and $B$ in Figure 1 is shown in Figure 8.

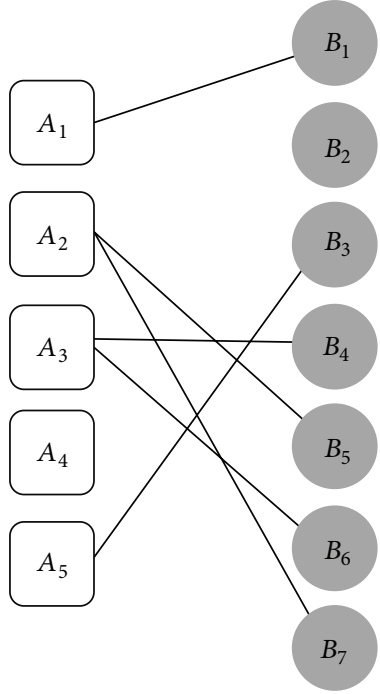

Figure 8: Final alignment between networks $A$ and $B$.

\section{Results}

In this section, we intend to compare our algorithm to other well-known local alignment algorithms (NetworkBLAST [9], MaWISh [10], Graemlin [11], and Graemlin 2.0 [13]), in a way that Graemlin 2.0 compares its results, because it is the only method that compares results of all local alignment tools against KEGG Ortholog (KO) groups [22] on different data sets. The data sets contain the PPI networks data from SNDB [21] for the organism pairs (E. coli, S. typhimurium) and (E. coli, C. crescentus), PPI networks data from DIP [20] for the organism pair (human, mouse), and data from IntAct [19] database for the organism pair (yeast, fly). The comparison is proceeded based on specificity and sensitivity in terms 
TABLE 1: Results of Pin-Align algorithm on four pairs of PPI networks, using different percentages in hub clustering step (Step 1).

\begin{tabular}{|c|c|c|c|c|c|}
\hline & Percentage & $C_{\mathrm{eq}}$ & $C_{\text {node }}$ & $C_{\text {or }}$ & Tot \\
\hline \multirow{4}{*}{$\begin{array}{l}\text { (E. coli, } S . \\
\text { typhimurium) }\end{array}$} & $60 \%$ & 0.95 & 0.93 & 1651 & 1779 \\
\hline & $70 \%$ & 0.95 & 0.94 & 1723 & 1835 \\
\hline & $80 \%$ & 0.95 & 0.94 & 1773 & 1890 \\
\hline & $90 \%$ & 0.95 & 0.94 & 1782 & 1905 \\
\hline \multirow{4}{*}{$\begin{array}{l}\text { (E. coli, C. } \\
\text { crescentus) }\end{array}$} & $60 \%$ & 0.87 & 0.84 & 666 & 794 \\
\hline & $70 \%$ & 0.89 & 0.86 & 665 & 772 \\
\hline & $80 \%$ & 0.88 & 0.85 & 693 & 811 \\
\hline & $90 \%$ & 0.89 & 0.87 & 764 & 876 \\
\hline \multirow{4}{*}{$\begin{array}{l}\text { (Human, } \\
\text { mouse) }\end{array}$} & $60 \%$ & 0.78 & 0.72 & 217 & 299 \\
\hline & $70 \%$ & 0.82 & 0.78 & 248 & 316 \\
\hline & $80 \%$ & 0.78 & 0.73 & 226 & 309 \\
\hline & $90 \%$ & 0.82 & 0.78 & 258 & 329 \\
\hline \multirow{4}{*}{ (Yeast, fly) } & $60 \%$ & 0.87 & 0.85 & 258 & 303 \\
\hline & $70 \%$ & 0.89 & 0.87 & 280 & 323 \\
\hline & $80 \%$ & 0.91 & 0.88 & 350 & 396 \\
\hline & $90 \%$ & 0.91 & 0.89 & 416 & 466 \\
\hline
\end{tabular}

TABLE 2: PPI networks from SNDB for the organism pairs (E. coli, S. typhimurium) and (E. coli, C. crescentus).

\begin{tabular}{|c|c|c|c|c|c|c|c|c|}
\hline & \multicolumn{4}{|c|}{ (E. coli, S. typhimurium) } & \multicolumn{4}{|c|}{ (E. coli, C. crescentus) } \\
\hline & $C_{\mathrm{eq}}$ & $C_{\text {node }}$ & $C_{\text {or }}$ & Tot & $C_{\mathrm{eq}}$ & $C_{\text {node }}$ & $C_{\text {or }}$ & Tot \\
\hline NB & 0.77 & 0.49 & 457 & 1016 & 0.78 & 0.50 & 346 & 697 \\
\hline Gr2.0 & 0.95 & 0.94 & 627 & 667 & 0.79 & 0.78 & 447 & 573 \\
\hline MW & 0.84 & 0.64 & 1309 & 2050 & 0.77 & 0.54 & 458 & 841 \\
\hline Gr2.0 & 0.97 & 0.96 & 1611 & 1678 & 0.77 & 0.76 & 553 & 727 \\
\hline $\mathrm{Gr}$ & 0.80 & 0.77 & 985 & 1286 & 0.69 & 0.64 & 546 & 847 \\
\hline Gr2.0 & 0.96 & 0.95 & 1157 & 1217 & 0.82 & 0.81 & 608 & 750 \\
\hline Pin-Align & 0.95 & 0.94 & 1782 & 1905 & 0.89 & 0.87 & 764 & 876 \\
\hline
\end{tabular}

of $\mathrm{KO}$ groups introduced in Graemlin 2.0 [13]. In shortas Graemlin 2.0 defined-to uniquely specify an alignment, the mapping should be transitive; that is, if protein $A$ is aligned with proteins $B$ and $C$, then protein $B$ must also be aligned with protein $C$. Mathematically, this means that the mapping is an equivalency relation, so groups of aligned proteins are referred to as equivalence classes. An equivalent class is defined as correct if all protein members in the class are in the same KO group. To measure specificity, Graemlin 2.0 computed two metrics:

(1) the fraction of equivalence classes that were correct $\left(C_{\text {eq }}\right)$,

(2) the fraction of nodes that were in correct equivalence classes $\left(C_{\text {node }}\right)$,

and to measure sensitivity, it computed two metrics:

(1) the total number of nodes that were in correct equivalence classes $\left(C_{\text {or }}\right)$,

(2) the number of equivalence classes that contained 2 species (Tot).
As we described in Step 1 of the Pin-Align algorithm, Pin-Align chooses some vertices as hubs whose degrees are greater than about $95 \%$ of total vertices. For networks $S$. typhimurium, C. crescentus, human, and yeast, Pin-Align chooses vertices as hubs in a way that their degrees are greater than $264,126,16$, and 23 , respectively. The reason why these degrees are greater than 95 percent of total vertices degrees is clear from cumulative frequency of their vertices degrees which is shown in charts of Figure 9. For example, in the PPI network of S. typhimurium the degrees of about $95 \%$ of vertices are less than 264, so in this network we choose vertices with degrees greater than 264 as hubs.

Table 1 shows Pin-Align results with different hub degrees. Column two of this table (percentage column) contains different percentages which are used for hub clustering step (Step 1).

The results of the algorithms are demonstrated in Tables 2 and 3. In these tables NB stands for NetworkBlast, MW for MaWISh, Gr for Graemlin, and Gr 2.0 for Graemlin 2.0. They show that Pin-Align is the most specific and sensitive aligner in comparison with other alignment tools like NetworkBlast, MaWISh, Graemlin, and Graemlin 2.0. Because Graemlin 2.0 
TABLE 3: PPI networks from DIP and IntAct for the organism pairs (human, mouse) and (yeast, fly).

\begin{tabular}{|c|c|c|c|c|c|c|c|c|}
\hline & \multicolumn{4}{|c|}{ (Human, mouse) } & \multicolumn{4}{|c|}{ (Yeast, fly) } \\
\hline & $C_{\mathrm{eq}}$ & $C_{\text {node }}$ & $C_{\text {or }}$ & Tot & $C_{\mathrm{eq}}$ & $C_{\text {node }}$ & $C_{\text {or }}$ & Tot \\
\hline NB & 0.33 & 0.06 & 65 & 1010 & 0.39 & 0.14 & 43 & 306 \\
\hline Gr2.0 & 0.83 & 0.81 & 228 & 281 & 0.58 & 0.58 & 155 & 267 \\
\hline MW & 0.59 & 0.36 & 87 & 241 & 0.45 & 0.37 & 10 & 27 \\
\hline Gr2.0 & 0.88 & 0.86 & 181 & 210 & 0.90 & 0.91 & 20 & 30 \\
\hline Gr & 0.59 & 0.53 & 108 & 203 & 0.33 & 0.29 & 35 & 122 \\
\hline Gr2.0 & 0.86 & 0.84 & 151 & 179 & 0.61 & 0.61 & 57 & 93 \\
\hline Pin-Align & 0.82 & 0.78 & 258 & 329 & 0.91 & 0.89 & 416 & 466 \\
\hline
\end{tabular}
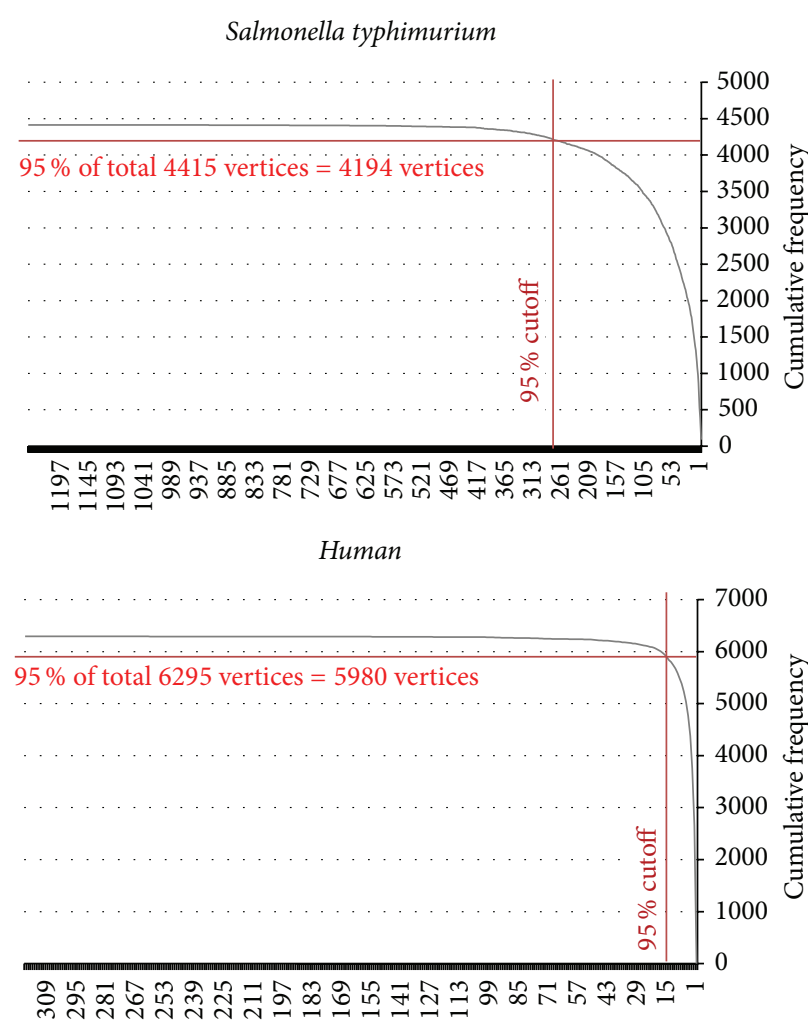

Caulobacter crescentus

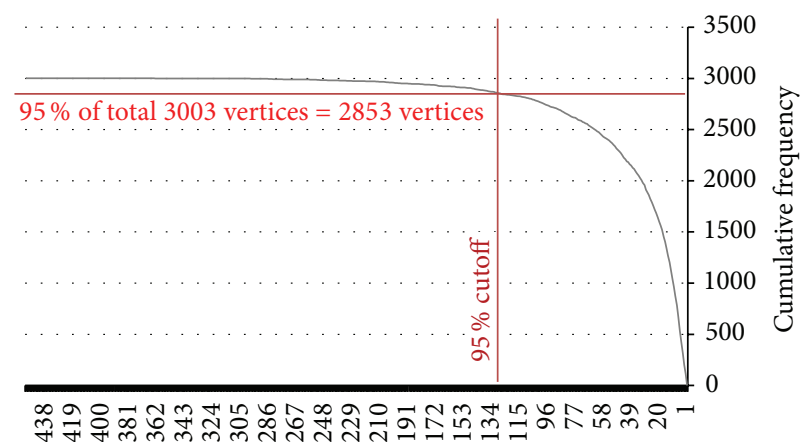

Yeast

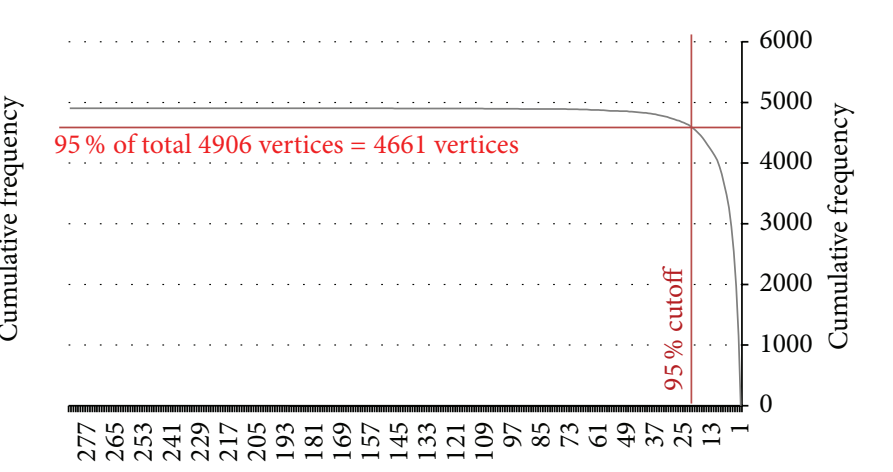

FIGURE 9: Cumulative frequency of vertices degrees in networks of S. typhimurium, C. crescentus, human, and yeast.

for each of its comparisons to other local aligner removed equivalence classes of its output that did not have a node in common with output of another local aligner, we have different results of Graemlin 2.0. Graemlin 2.0 did this due to considerations of local aligners to nodes in conserved modules and global aligners to all nodes.

In Table 2, we use the PPI network data from SNDB for the organism pairs (E. coli, S. typhimurium) and (E. coli, C. crescentus). In Table 3 we use the PPI networks data from DIP and IntAct for the organism pairs (human, mouse) and (yeast, fly).

Figure 10 shows that Pin-Align also finds more correct equivalence classes than NetworkBlast, MaWISh, Graemlin, and Graemlin 2.0. By considering all the above results and comparing Pin-Align to the other algorithms, it is evident that Pin-Align is more accurate in most cases based on the $C_{\text {eq }}$, $C_{\text {node }}, C_{\text {or }}$, and Tot measures.

\section{Conclusions}

In this paper, we presented Pin-Align, a pairwise local network alignment to improve accuracy of the alignment. Pin-Align algorithm is mainly designed based on the dynamic programming approach and has specificity and sensitivity comparable with existing tools such as NetworkBlast, Graemlin, Graemlin 2.0, and MaWISh. Our novel scoring system is based on bridges which are considerable edges due to the 

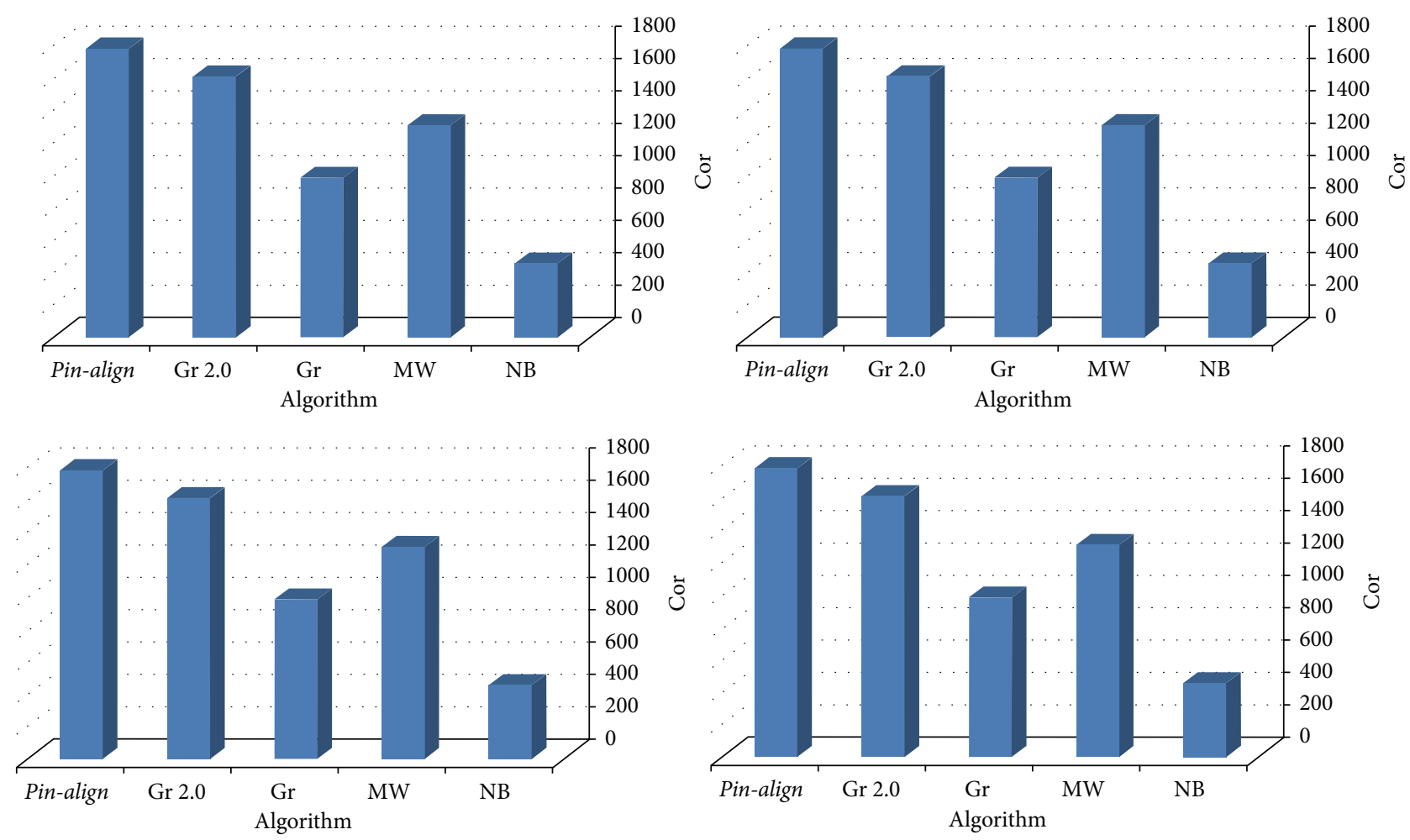

Figure 10: The number of correct equivalence classes that are found in alignment of organism pairs (E. coli, S. typhimurium), (E. coli, C. crescentus), (human, mouse), and (yeast, fly) by Pin-Align, in comparison with Graemlin 2.0, Graemlin, MaWISh, and NetworkBlast.

existence of hubs and small words in biological networks which certainly contain bridges. Bridges take part in most of the paths and consequently conserved paths in the networks. In future work, we plan to extend this approach to multiple network alignment.

\section{Conflict of Interests}

The authors declare that there is no conflict of interests regarding the publication of this paper.

\section{Acknowledgments}

The authors would like to thank Mehdi Sadeghi for valuable comments and suggestions and Graemlin team [13] from Stanford University for sharing the datasets and helpful communication. The authors would like to acknowledge the financial support of University of Tehran for this research under Grant no. 6151184/01/04.

\section{References}

[1] O. Kuchaiev, T. Milenković, V. Memišević, W. Hayes, and N. Pržulj, "Topological network alignment uncovers biological function and phylogeny," Journal of the Royal Society Interface, vol. 7, no. 50, pp. 1341-1354, 2010.

[2] Y. Wang, T. Hindemitt, and K. F. X. Mayer, "Significant sequence similarities in promoters and precursors of Arabidopsis thaliana non-conserved microRNAs," Bioinformatics, vol. 22, no. 21, pp. 2585-2589, 2006.

[3] P.-A. He, X.-F. Li, J.-L. Yang, and J. Wang, "A novel descriptor for protein similarity analysis," MATCH Communications in Mathematical and in Computer Chemistry, vol. 65, no. 2, pp. 445-458, 2011.

[4] Y. Zhang and W. Chen, "A new measure for similarity searching in DNA sequences," MATCH Communications in Mathematical and in Computer Chemistry, vol. 65, no. 2, pp. 477-488, 2011.

[5] T. Milenković and N. Pržulj, "Uncovering biological network function via graphlet degree signatures," Cancer Informatics, vol. 6, pp. 257-273, 2008.

[6] T. Milenković, W. L. Ng, W. Hayes, and N. Pržulj, "Optimal network alignment with graphlet degree vectors," Cancer Informatics, vol. 9, pp. 121-137, 2010.

[7] B. Neyshabur, A. Khadem, S. Hashemifar, and S. S. Arab, "NETAL: a new graph-based method for global alignment of protein-protein interaction networks," Bioinformatics, vol. 29, no. 13, pp. 1654-1662, 2013.

[8] B. P. Kelley, B. Yuan, F. Lewitter, R. Sharan, B. R. Stockwell, and T. Ideker, "PathBLAST: a tool for alignment of protein interaction networks," Nucleic Acids Research, vol. 32, pp. 83$88,2004$.

[9] R. Sharan, S. Suthram, R. M. Kelley et al., "Conserved patterns of protein interaction in multiple species," Proceedings of the National Academy of Sciences of the United States of America, vol. 102, no. 6, pp. 1974-1979, 2005.

[10] M. Koyuturk, Y. Kim, U. Topkara, S. Subramaniam, W. Szpankowski, and A. Grama, "Pairwise alignment of protein 
interaction networks," Journal of Computational Biology, vol. 13, no. 2, pp. 182-199, 2006.

[11] J. Flannick, A. Novak, B. S. Srinivasan, H. H. McAdams, and S. Batzoglou, "Græmlin: general and robust alignment of multiple large interaction networks," Genome Research, vol. 16, no. 9, pp. 1169-1181, 2006.

[12] R. Singh, J. Xu, and B. Berger, "Global alignment of multiple protein interaction networks," in Proceedings of the 13th Pacific Symposium on Biocomputing (PSB '08), pp. 303-314, Kohala Coast, Hawaii, USA, January 2008.

[13] J. Flannick, A. Novak, C. B. Do, B. S. Srinivasan, and S. Batzoglou, "Automatic parameter learning for multiple local network alignment," Journal of Computational Biology, vol. 16, no. 8, pp. 1001-1022, 2009.

[14] C.-S. Liao, K. Lu, M. Baym, R. Singh, and B. Berger, "IsoRankN: spectral methods for global alignment of multiple protein networks," Bioinformatics, vol. 25, no. 12, pp. i253-i258, 2009.

[15] R. Andersen, F. Chung, and K. Lang, "Local graph partitioning using PageRank vectors," in Proceedings of the 47th Annual IEEE Symposium on Foundations of Computer Science (FOCS '06), pp. 475-486, IEEE Computer Society, Los Alamitos, Calif, USA, October 2006.

[16] W. Tian and N. F. Samatova, "Pairwise alignment of interaction networks by fast identification of maximal conserved patterns," Pacific Symposium on Biocomputing, vol. 14, pp. 99-110, 2009.

[17] W. Tian and N. F. Samatova, "Global alignment of pairwise protein interaction networks for maximal common conserved patterns," International Journal of Genomics, vol. 2013, Article ID 670623, 11 pages, 2013.

[18] S. F. Altschul, T. L. Madden, A. A. Schäffer et al., "Gapped BLAST and PSI-BLAST: a new generation of protein database search programs," Nucleic Acids Research, vol. 25, no. 17, pp. 3389-3402, 1997.

[19] S. Kerrien, Y. Alam-Faruque, B. Aranda et al., "IntAct-Open source resource for molecular interaction data," Nucleic Acids Research, vol. 35, no. 1, pp. D561-D565, 2007.

[20] I. Xenarios, Ł. Salwínski, X. J. Duan, P. Higney, S.-M. Kim, and D. Eisenberg, "DIP, the Database of Interacting Proteins: a research tool for studying cellular networks of protein interactions," Nucleic Acids Research, vol. 30, no. 1, pp. 303-305, 2002.

[21] B. S. Srinivasan, A. F. Novak, J. A. Flannick, S. Batzoglou, and H. H. McAdams, "Integrated protein interaction networks for 11 microbes," in Research in Computational Molecular Biology, vol. 3909 of Lecture Notes in Computer Science, pp. 1-14, 2006.

[22] M. Kanehisa, S. Goto, Y. Sato, M. Kawashima, M. Furumichi, and M. Tanabe, "Data, information, knowledge and principle: back to metabolism in KEGG," Nucleic Acids Research, vol. 42, no. 1, pp. D199-D205, 2014.

[23] R. Singh, J. Xu, and B. Berger, "Pairwise global alignment of protein interaction networks by matching neighborhood topology," in Proceedings of the 11th Annual International Conference on Research in Computational Molecular Biology (RECOMB '07), pp. 16-31, April 2007.

[24] T. H. Cormen, C. E. Leiserson, R. L. Rivest, and S. Clifford, Introduction to Algorithms, MIT Press, Cambridge, UK, 3rd edition, 2009.

[25] S. E. Schaeffer, "Graph clustering," Computer Science Review, vol. 1, no. 1, pp. 27-64, 2007.

[26] A. Konak, D. W. Coit, and A. E. Smith, "Multi-objective optimization using genetic algorithms: a tutorial," Reliability Engineering and System Safety, vol. 91, no. 9, pp. 992-1007, 2006.
[27] H. W. Kuhn, "The Hungarian method for the assignment problem," Naval Research Logistics, vol. 2, pp. 83-97, 1955. 


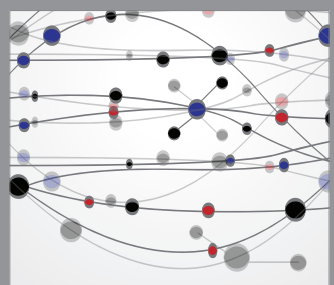

The Scientific World Journal
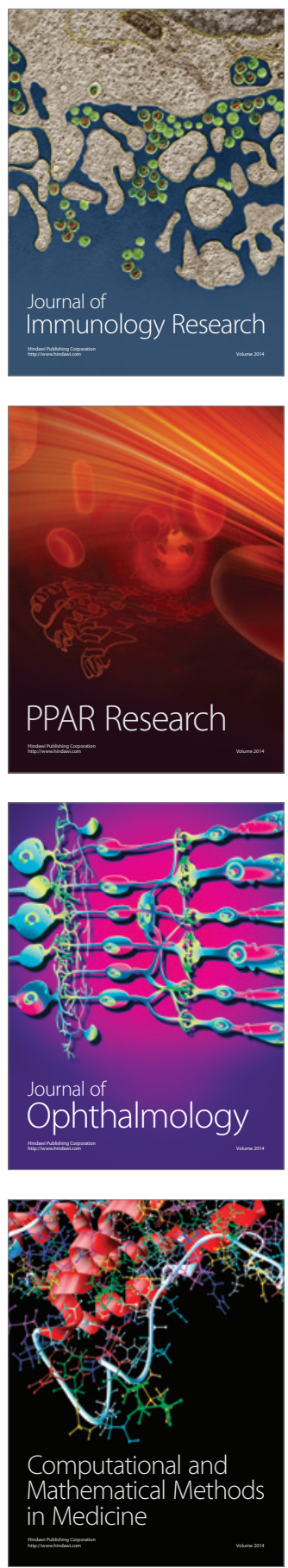

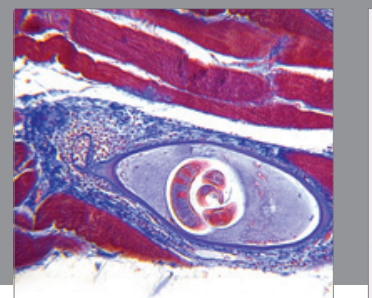

Gastroenterology

Research and Practice
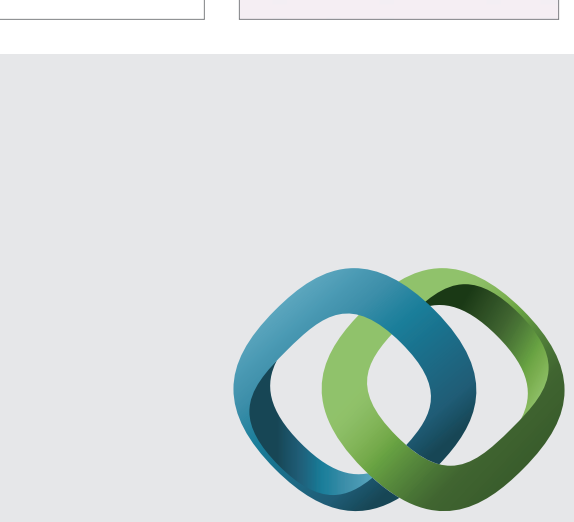

\section{Hindawi}

Submit your manuscripts at

http://www.hindawi.com
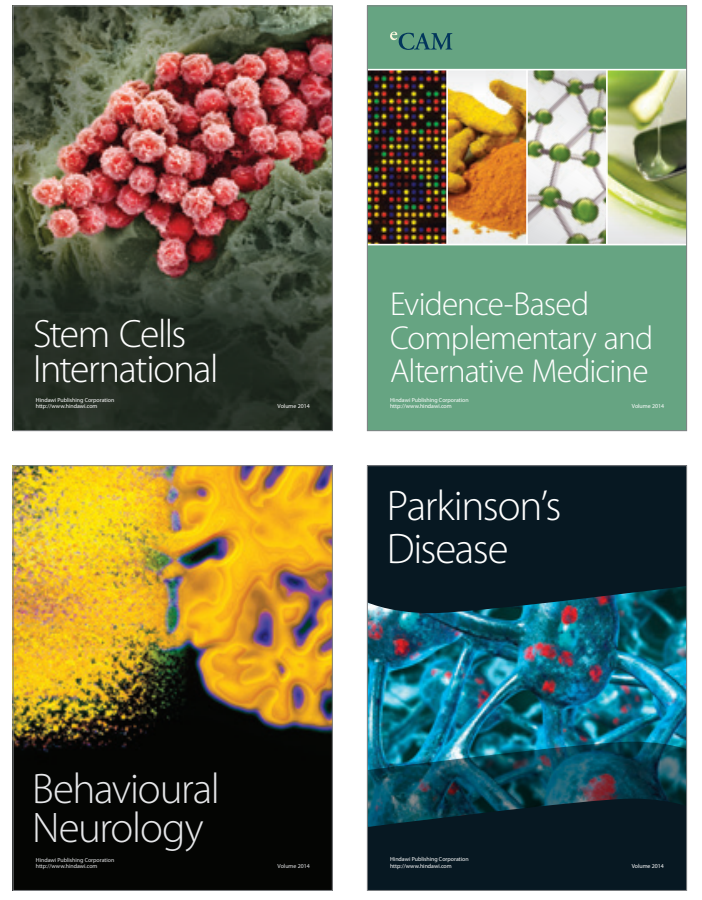
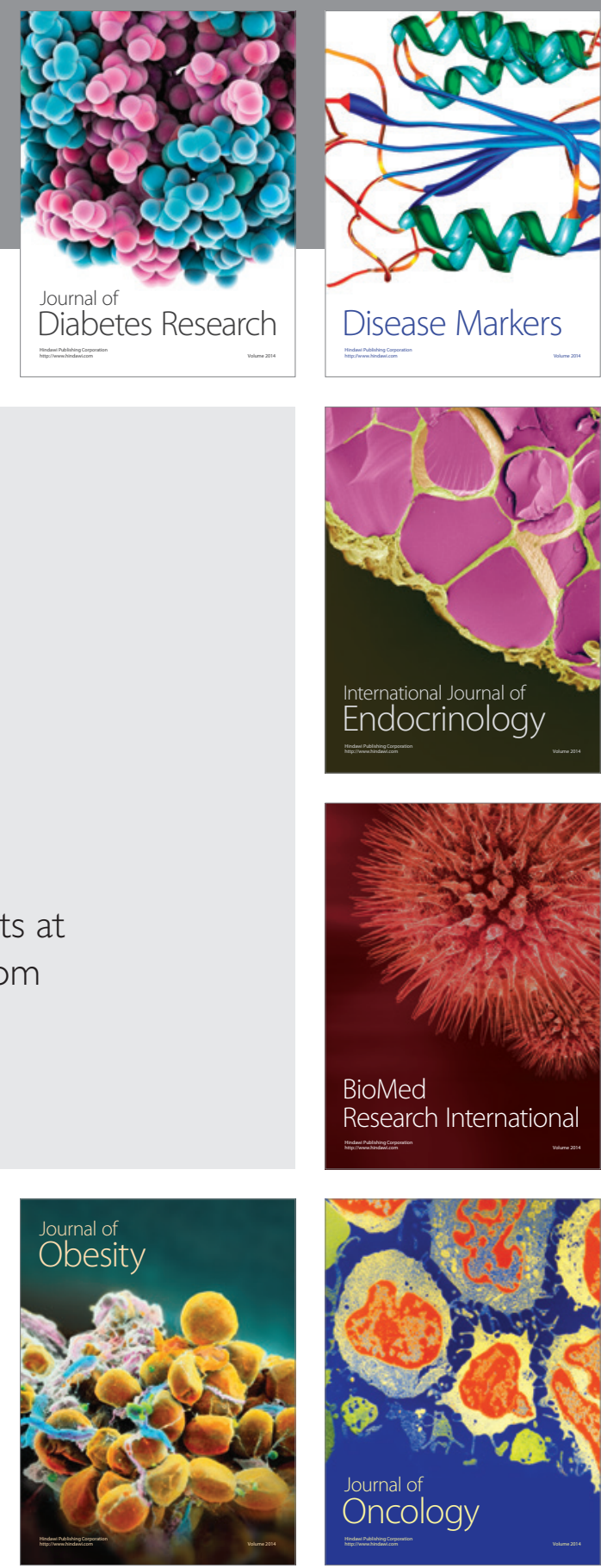

Disease Markers
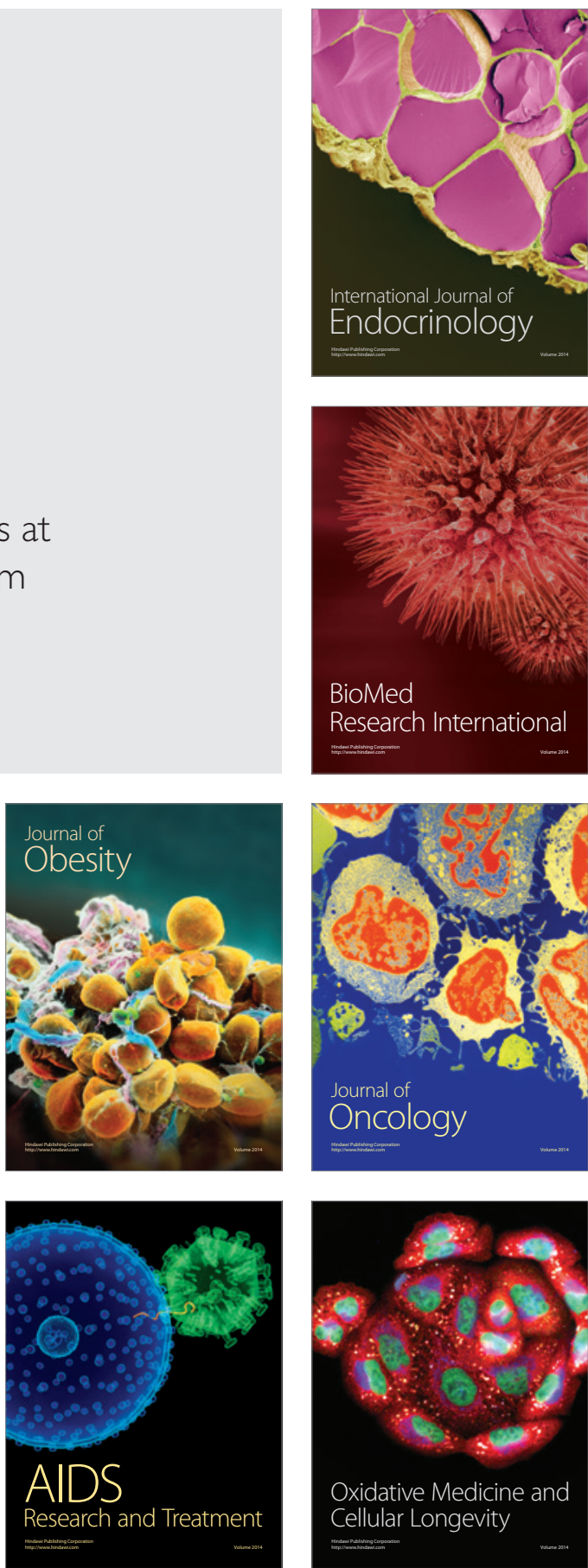